\title{
Efeitos do canabidiol nos sinais e comorbidades do transtorno do espectro autista
}

\author{
Effects of cannabidiol on the signs and comorbidities of autistic spectrum disorder \\ Efectos del cannabidiol sobre los signos y las comorbilidades del trastorno del espectro autista
}

Recebido: 22/07/2021 | Revisado: 28/07/2021 | Aceito: 29/07/2021 | Publicado: 05/08/2021

\author{
Flávia Cristina Osaku Minella \\ ORCID: https://orcid.org/0000-0001-7694-3791 \\ Centro Universitário da Fundação Assis Gurgacz, Brasil \\ E-mail: fcominella@minha.fag.edu.br \\ Vagner Fagnani Linartevichi \\ ORCID: https://orcid.org/0000-0002-2624-7744 \\ Centro Universitário da Fundação Assis Gurgacz, Brasil \\ E-mail: linartevichi@fag.edu.br
}

\begin{abstract}
Resumo
O autismo é considerado um transtorno neuropsiquiátrico, com desenvolvimento na infância precoce, havendo prejuízos nos seguintes domínios: interação social, comunicação, interesses restritos e padrões estereotipados do comportamento. Não há tratamento definitivo estabelecido para o autismo, sendo o objetivo das intervenções farmacológicas o alívio de sinais específicos. Em geral, os medicamentos tradicionalmente utilizados (antipsicóticos atípicos, inibidores seletivos de recaptação de serotonina, estimulantes e anticonvulsivantes) apresentam diversos efeitos colaterais, refletindo em descontinuidade do tratamento. O canabidiol (CBD), componente da planta Cannabis Sativa, é livre dos efeitos lisérgicos e tem sido utilizado como uma alternativa ao tratamento do Transtorno do Espectro Autista (TEA). O sistema endocaninóide atua em diversas funções fisiológicas, estando o potencial terapêutico do CBD na regulação das funções hiper ou hipoestimuladas. Desta forma, foi elaborada uma revisão sistemática dos estudos clínicos que utilizaram o CBD no tratamento dos sinais e comorbidade do TEA. As bases de dados utilizadas foram PubMed e Medline, sendo considerados os seguintes descritores em cada base: autism and treatment and cannabis e autism and treatment and cannabidiol, no título e resumo. Foram incluídas pesquisas publicadas em periódicos nacionais e internacionais, nos idiomas inglês e português, com livre acesso e distribuídos na integra. Os artigos selecionados foram lidos na íntegra e extraídos dados relevantes para análise e interpretação. Os resultados mostram que de forma geral, os participantes dos ensaios-clínicos mostraram ter melhoras de todos os sinais associados ao TEA considerados em cada estudo.
\end{abstract}

Palavras-chave: Transtorno do espectro autista; Tratamento; Canabidiol; Cannabis.

\begin{abstract}
Autism is considered a neuropsychiatric disorder, with development in early childhood, with losses in the following domains: social interaction, communication, restricted interests and stereotyped patterns of behavior. There is no established definitive treatment for autism, and the objective of pharmacological ones is the relief of specific signs. In general, traditionally used medications (atypical antipsychotics, selective serotonin reuptake inhibitors, stimulants and anticonvulsants) have several side effects, reflecting treatment discontinuation. Cannabidiol (CBD), a component of the Cannabis Sativa plant, is free from lysergic effects and has been used as an alternative to the treatment of Autistic Spectrum Disorder (ASD). The endocaninoid system acts in several physiological functions, accessing the therapeutic potential of CBD in the regulation of hyper or hypostimulated functions. Thus, a systematic review of clinical studies that used CBD in the treatment of signs and comorbidity of ASD was prepared. The databases used were PubMed and Medline, considering the following descriptors in each database: "autismo" and "treatment" and "cannabis"; and "autismo" and "treatment" and "canabidiol", in the title and abstract. Manuscripts must be published in national and international journals, in English and Portuguese, and free full text. The selected manuscripts were read in full and relevant data extracted for analysis and interpretation. The results presented that overall, clinical trial participants have been shown to have improvements in all signs associated with the ASD considered in each study.
\end{abstract}

Keywords: Autistic spectrum disorder; Treatment; Canabidiol; Cannabis.

\section{Resumen}

El autismo es considerado un trastorno neuropsiquiátrico, con desarrollo en la primera infancia, con pérdidas en los siguientes dominios: interacción social, comunicación, intereses restringidos y patrones de comportamiento estereotipados. No existe un tratamiento definitivo establecido para el autismo, siendo el objetivo de las intervenciones farmacológicas el alivio de signos específicos. En general, los medicamentos de uso tradicional (antipsicóticos atípicos, inhibidores selectivos de la recaptación de serotonina, estimulantes y anticonvulsivos) tienen varios efectos secundarios que reflejan la interrupción del tratamiento. El cannabidiol (CBD), un componente de la 
planta Cannabis Sativa, no tiene efectos lisérgicos y se ha utilizado como alternativa al tratamiento del trastorno del espectro autista (TEA). El sistema endocaninoide actúa en varias funciones fisiológicas, siendo el potencial terapéutico del CBD en la regulación de funciones hiper o hipoestimuladas. Por lo tanto, se elaboró una revisión sistemática de los estudios clínicos que utilizaron CBD en el tratamiento de los signos y la comorbilidad del TEA. Las bases de datos utilizadas fueron PubMed y Medline, considerándose en cada base de datos los siguientes descriptores: "autismo" y "tratamento" y "cannabis"; y "autismo" y "tratamento" y "canabidiol", en el título y resumen. Se incluyeron investigaciones publicadas en revistas nacionales e internacionales, en inglés y portugués, de libre acceso y distribuidas íntegramente. Los artículos seleccionados se leyeron en su totalidad y se extrajeron los datos relevantes para su análisis e interpretación. Los resultados muestran que, en general, los participantes de los ensayos clínicos mostraron una mejoría en todos los signos asociados con el TEA considerados en cada estudio.

Palabras clave: Trastorno del espectro autista; Tratamento; Canabidiol; Cannabis.

\section{Introdução}

A etiologia do autismo é heterogênea, incluindo fatores genéticos, ambientais e mecanismos epigenéticos (Arberas \& Ruggieri, 2019; Oliveira \& Souza, 2021; Reece \& Hulse, 2019). O termo “distúrbio autístico do contato afetivo" foi criado por Kanner em 1943; no ano seguinte, Hans Asperger descreveu a dificuldade de criança autistas no estabelecimento de laços afetivos e no diálogo. Nas décadas de 50 e 60, o autismo era classificado como um subgrupo da esquizofrenia, resultando em tratamentos ineficazes; passando a ser considerado, a partir das pesquisas de Rutter na década de 70, como um transtorno cognitivo. O autismo como espectro denominado Síndrome de Asperger foi estabelecido por Wing em 1981 (Silva, et al., 2020).

Segundo Mottron e Bzdok (2020), há um aumento de 20 vezes na prevalência relatada do TEA nos últimos 30 anos nos Estados Unidos, em parte devido a fragmentação do fenótipo do autismo em "traços autistas", que resultam de critérios diagnósticos não específicos.

O autismo é considerado como um transtorno neuropsiquiátrico, com desenvolvimento na infância precoce, fazendo parte dos chamados transtornos invasivos do desenvolvimento (TIDs), ou transtornos do "espectro do autismo". São percebidos prejuízos, evidenciados principalmente a partir dos três anos de idade, em diversas áreas do funcionamento, atrelado muitas vezes a atrasos no desenvolvimento mental (Rubim \& Hora, 2020).

Para o diagnóstico três domínios devem estar afetados, a saber: 1) interação social; 2) comunicação; 3) interesses restritos e padrões estereotipados do comportamento (incluindo movimentos estereotipados e maneirismos). A interação social é afetada pelas dificuldades na comunicação verbal e não-verbal, ou mesmo falta de interesse em se comunicar. Os déficits em tais domínios podem levar a incapacitação do indivíduo afetado (Silva \& Coelho, 2021).

O tratamento do TEA deve ser individualizado e estar em consonância com o nível de comprometimento da patologia. Porém, não há tratamento definitivo estabelecido, ou mesmo tratamentos medicamentosos aprovados pelo FDA (Food and Drug Administration, dos EUA) para o autismo. De forma geral, as intervenções farmacológicas visam aliviar sinais específicos. Tradicionalmente, são utilizados medicamentos de diferentes grupos, não sendo bem esclarecidos os benefícios de longo prazo, fator que reflete na descontinuidade do tratamento por grande parte dos pacientes; parte pela percepção da perda da eficácia do agente farmacológico utilizado, parte pelos efeitos colaterais. As categorias de fármacos comumente utilizados no autismo abarcam os antipsicóticos atípicos (AAPs) e os inibidores seletivos de recaptação de serotonina (ISRSs), como também os medicamentos estimulantes e anticonvulsivantes (Nunes, et al., 2021).

Os antipsicóticos atípicos desenvolvidos originalmente para o tratamento da psicose, incluem: a clozapina, a risperidona, a olanzapina, a quetiapina, a ziprazidona e o aripiprazol. Tais agentes farmacológicos são amplamente utilizados no tratamento de comportamentos mal adaptativos do TEA, como: automutilação, episódio de agressão e crises de ira. Em relação aos antipsicóticos típicos, como o haloperidol, os AAPs oferecem vantagens que incluem, em curto prazo, menos efeitos colaterais e, em longo prazo, discinesia tardia. Ainda, há a melhora de sinais "negativos" da esquizofrenia e das 
estereotipias, que impactam nas relações sociais dos indivíduos (Maneeton, et al., 2018). O mecanismo de ação é dado pela ação da 5-hidroxitriptamina (5-HT) no bloqueio do receptor de dopamina (DA) e, possivelmente, por haver um deslocamento das AAPs pela DA endógena no estriado, uma vez que não há um vínculo forte aos receptores pós-sinápticos dopaminérgicos (Nikolov, Jonker, \& Scahill, 2006).

Já o mecanismo de ação dos inibidores da recaptação da serotonina (IRSs), com significativa aplicabilidade clínica no TEA, tais como: fluoxetina, fluvoxamina, sertralina, citalopram e mirtazapina; está baseado na inibição da recaptação da serotonina no sítio do transportador pré-sináptico. Os estimulantes, como o metilfenidato, atuam nas atividades comórbidas e mostraram benefícios no tratamento de sinais como: agitação motora, hiperatividade, distratibilidade e comportamento disruptivo (Reddihough, et al., 2019). Os anticonvulsivantes, como divalproato sódico também podem trazer melhoras da instabilidade, e dos comportamentos repetitivos e agressão (Hollander, et al., 2001).

Uma alternativa para redução dos sinais dos pacientes com TEA, com aumento na frequência do seu uso nos últimos anos, são os preparados a base de canabidiol (CBD) (Pretzsch, et al., 2019), segundo componente mais abundante da Cannabis Sativa e livre dos efeitos lisérgicos.

Pesquisas apontam (Devinsky, et al., 2014; Starowicz \& Finn, 2017; Kis, et al., 2019; Cooray, et al., 2020; FerreiraJunior, et al., 2020) efeitos positivos desse princípio ativo no tratamento da doença de Parkinson, Alhzeimer, epilepsia refratária aos tratamentos convencionais, tratamento paliativo do câncer e dor crônica (Linartevichi, Cury, \& Nascimento, 2021). No TEA, pondera-se ainda o CBD como tratamento adjuvante de crise convulsiva.

O sistema endocanabinoide atua na moderação das funções fisiológicas, como na regulação do apetite, sono, dor, sistema imunológico, entre outros (Pacher \& Kunos, 2013). O receptor CB1 encontrado na medula espinhal e no cérebro, e o CB2 encontrado nas células do sistema imune e nervoso, são os dois principais receptores dos canabinoides, seja eles endógenos ou exógenos como da Cannabis Sativa. O potencial terapêutico do CBD está na regulação das funções hiper ou hipoestimuladas. Segundo Chakrabarti et al. (2015), há evidências que comprovam que o sistema endocanabinoide atuam em quatro características fenotípicas do autismo: 1) responsividade de recompensa social, 2) desenvolvimento neural, 3) ritmo circadiano, e 4) sintomas relacionados à ansiedade; havendo campos para exploração terapêutica de elementos distintos desse sistema.

O objetivo deste estudo foi realizar uma revisão sistemática da literatura de estudos que tenham incluído o canabidiol no tratamento do Transtorno do Espectro Autista (TEA), possibilitando a avaliação dos efeitos do canabidiol na redução dos sinais e comorbidades do TEA.

\section{Metodologia}

Para a revisão sistemática de abordagem qualitativa (Estrela, 2018) foram utilizadas as bases de dados PubMed e Medline, sendo considerados os seguintes descritores em cada base: autism and treatment and cannabis e autism and treatment and cannabidiol, no título e resumo.

Os critérios de inclusão foram pesquisas publicadas em periódicos nacionais e internacionais, nos idiomas inglês e português, indexados nas bases de dado anteriormente citadas, com livre acesso e distribuídos na integra. Optou-se por não realizar recorte temporal. Foram excluídos artigos de revisão.

Os títulos e resumos identificados na busca inicial foram avaliados por dois pesquisadores de forma independente. As discordâncias entre os pesquisadores foram resolvidas por consenso. O fluxograma da etapa de seleção dos artigos consta na Figura 1. 
Figura 1: Fluxograma da etapa de seleção de artigos.

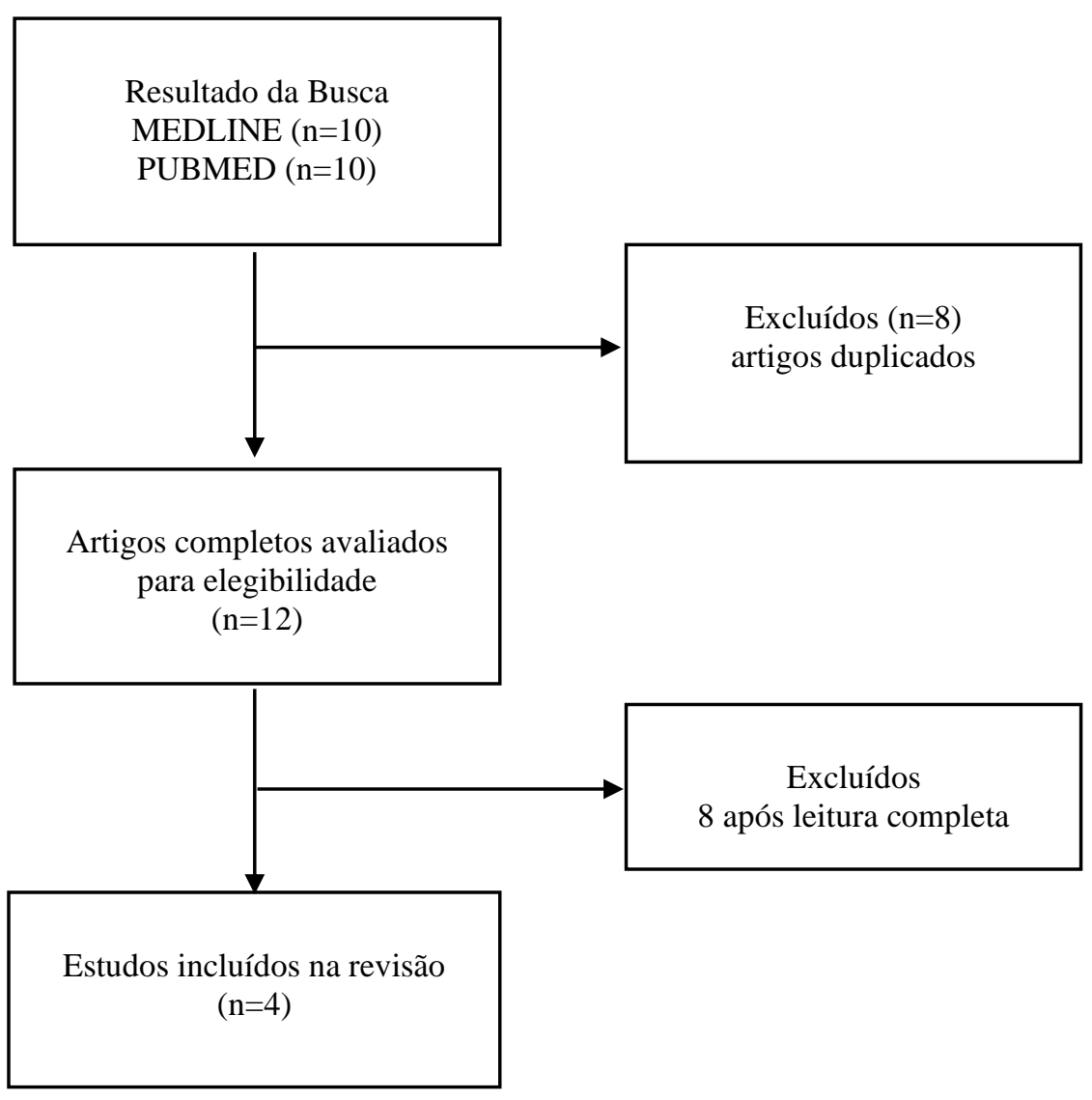

Fonte: Autores.

Para as duas combinações de palavras-chaves, excluindo-se artigos repetidos entre tais combinações, foram encontrados 10 artigos no Medline e 10 artigos no PubMed. Do total desses 20 artigos, oito eram repetidos entre as bases de dados, restando 12 artigos, dos quais quatro atenderam os critérios de elegibilidade após leitura dos resumos. Os artigos selecionados foram lidos na íntegra e extraídos dados relevantes para análise e interpretação.

\section{Resultados}

De forma geral, nos quatro artigos selecionados (Tabela 1), como instrumento para análise da melhora dos sintomas, os pesquisadores aplicaram questionários estruturados ou entrevistas aos pais/cuidadores, sendo de natureza observacional. Em todos os casos, os dados foram avaliados por um grupo independente e multidisciplinar de especialistas.

Barchel, et al. (2018) analisaram os efeitos do canabidiol em 53 pacientes com idade média de 11 anos (85\% do sexo masculino e $15 \%$ do sexo feminino), acompanhados pelo período mínimo de 30 dias após o início do tratamento (duração média de 66 dias). A partir do uso da solução de óleo canabinóide na concentração de 30\% e proporção de CBD/THC de 1/20, houve uma melhora de: 68,4\% no sintoma de hiperatividade; 67,6\% no comportamento autolesivo; 71,4\% em problemas de sono; por fim, 47,1\% nos sinais de ansiedade. Em todos as categorias de sinais, não houve inferioridade do CBD em relação aos tratamentos convencionais. Considerando a mudança geral nos sinais associados ao TEA, houve uma melhora em 74,5\%, nenhuma alteração em $21,6 \%$ e piora em 3,9\%. Os efeitos colaterais foram relatados como transitórios e sem repercussões. 
Tabela 1 - Estudos selecionados para a revisão sistemática do uso de CBD no TEA.

\begin{tabular}{|c|c|c|c|c|}
\hline Autores / Ano & Concentração / Dosagem & Período de tratamento & População & $\begin{array}{l}\text { Percepção de melhora dos } \\
\text { sinais }\end{array}$ \\
\hline $\begin{array}{l}\text { Barchel et al. } \\
(2018)\end{array}$ & $\begin{array}{l}\text { Concentração de } 30 \% \text {, } \\
\text { proporção de } 1 / 20 \mathrm{de} \\
\text { canabidiol (CBD)/ } \Delta 9 \text { - } \\
\text { tetrahidrocanabinol (THC) } \\
\text { Dose diária recomendada: } \\
\text { - } \quad \text { CBD: } 16 \mathrm{mg} / \mathrm{kg} \\
\text { (dose diária } \\
\text { máxima de } 600 \\
\text { mg) } \\
\text { THC - dose diária } \\
\text { de } 0,8 \mathrm{mg} / \mathrm{kg} \\
\text { (dose diária } \\
\text { máxima de } 40 \\
\text { mg) }\end{array}$ & 30 dias a 588 dias & $\begin{array}{l}n=53 \\
\text { Idade média: } 11 \text { anos } \\
(4-22)\end{array}$ & $\begin{array}{l}\text { Hiperatividade }(n=38) \text { : } \\
\text { melhora em } 68,4 \% \text {; sem } \\
\text { alterações em } 28,9 \% \text { e } \\
\text { piora em } 2,6 \% \text {. } \\
\text { Comportamento } \\
\text { autolesivo }(n=34): \\
\text { melhora em } 67,6 \% \text { e } \\
\text { piora em } 8,8 \% \text {. } \\
\text { Problemas de sono } \\
\text { ( } n=21) \text { : melhorar em } \\
71,4 \% \text { e piora em } 4,7 \% . \\
\text { Ansiedade }(n=17): \\
\text { melhora em } 47,1 \% \text { e } \\
\text { piora em } 23,5 \% .\end{array}$ \\
\hline $\begin{array}{l}\text { Fleury- } \\
\text { Teixeira et al. } \\
(2019)\end{array}$ & $\begin{array}{l}\text { Extrato de CBD, proporção } \\
\text { de 75/1 de CBD/THC } \\
\text { Dose média diária: } \\
\text { • CBD: } 4,55 \mathrm{mg} / \mathrm{kg} \\
\text { (dose diária } \\
\text { máxima de } 6,45 \\
\text { mg/kg/dia) } \\
\text { THC - dose diária } \\
\text { de } 0,06 \quad \mathrm{mg} / \mathrm{kg} \\
\text { (dose diária } \\
\text { máxima de } 0,09 \\
\text { mg/kg/dia) }\end{array}$ & 6 a 9 meses & $\begin{array}{l}n \text { inicial }=18 \\
n \text { considerado }=15 \\
\text { Idade média: } 10,9 \text { anos } \\
(7-18)\end{array}$ & 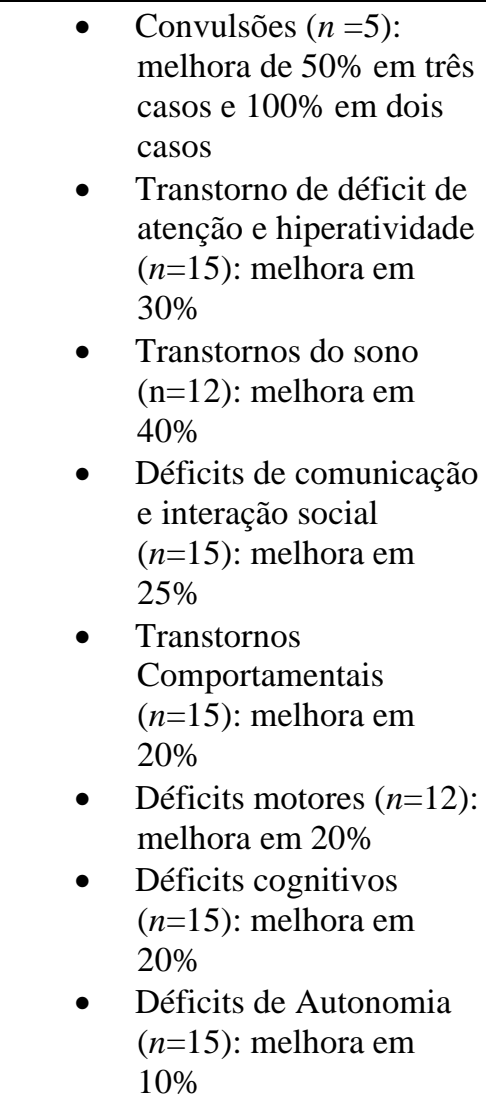 \\
\hline
\end{tabular}




\begin{tabular}{|c|c|c|c|c|}
\hline $\begin{array}{l}\text { Schleider et al. } \\
\text { (2019) }\end{array}$ & $\begin{array}{l}30 \% \text { de CBD e } 1,5 \% \text { de } \\
\text { THC } \\
\text { Dose média - três vezes ao } \\
\text { dia: } \\
\text { - CBD: } 79,5 \pm 61,5 \\
\text { mg } \\
\text { - THC: } 4,0 \pm 3,0 \\
\quad \text { mg }\end{array}$ & & $\begin{array}{l}n \text { inicial }=188 \\
n \text { após } 1 \text { mês }=179 \\
n \text { após } 6 \text { meses }=155 \\
\begin{array}{l}\text { Idade média: } 12,9 \text { anos } \\
\text { (menos de } 5-18 \text { ) }\end{array}\end{array}$ & $\begin{array}{l}\text { Boa qualidade de vida: } \\
\text { melhora em } 35,5 \% \\
\text { - } \quad \text { Humor positivo: } \\
\text { melhora em } 21,5 \% \\
\text { - } \quad \text { Capacidade de se vestir } \\
\text { e tomar banho de forma } \\
\text { independente: melhora } \\
\text { em } 16,5 \% \\
\text { - } \quad \text { Bom sono: melhora em } \\
\text { 21,4\% } \\
\text { Boa concentração: } \\
\text { melhora em } 14 \% \\
\text { - Convulsões: } 84,6 \% \\
\text { desaparecimento dos } \\
\text { sinais } \\
\text { Inquietação: melhora em } \\
\text { 91\% } \\
\text { Ataques de raiva: } \\
\text { melhora em } 90,3 \%\end{array}$ \\
\hline $\begin{array}{l}\text { Aran et al. } \\
(2021)\end{array}$ & $\begin{array}{l}\text { Teste 1: } \\
\text { Extrato da Cannabis } \\
\text { Proporção de } 20 / 1 \text { de CBD } \\
\text { e THC } \\
\text { Teste } 2 \text { : } \\
\text { CBD puro e THC puro na } \\
\text { mesma proporção } \\
\text { Dose inicial: } \\
\text { CBD: } 1 \mathrm{mg} / \mathrm{kg} \\
\text { THC: } 0,05 \mathrm{mg} / \mathrm{kg} \\
\text { A dose foi aumentada em } \\
\text { dias alternados em } 1 \mathrm{mg} / \mathrm{kg} \\
\text { de CDB e } 0,05 \mathrm{mg} / \mathrm{kg} \mathrm{de} \\
\text { THC até atingir: } \\
\text { para crianças com peso de } \\
20-40 \mathrm{~kg}: \\
\text { CBD: } 10 \mathrm{mg} / \mathrm{kg} \\
\text { THC: } 0,5 \mathrm{mg} / \mathrm{kg} \text {, ou } \\
\text { para crianças com peso de } \\
>40 \mathrm{~kg}: \\
\text { CBD: } 7,5 \mathrm{mg} / \mathrm{kg} \\
\text { THC: } 0,375 \mathrm{mg} / \mathrm{kg} \\
\text { (até um máximo de } 420 \mathrm{mg} \\
\text { de CBD e } 21 \mathrm{mg} \mathrm{de} \mathrm{THC} \\
\text { por dia), dividido em } 3 \\
\text { doses diárias }\end{array}$ & $\begin{array}{l}2 \text { semanas (teste de } \\
\text { eficácia) + } 4 \text { semanas } \\
\text { (wash-out) }+12 \\
\text { semanas (avaliação da } \\
\text { tolerabilidade) }\end{array}$ & $\begin{array}{l}n=150 \\
\text { Idade média: } 11,8 \text { anos } \\
(5-21)\end{array}$ & $\begin{array}{l}\text { - Comportamento } \\
\text { disruptivo: melhora em } \\
49 \% \text { no extrato da } \\
\text { planta inteira }(n=45) \\
\text { versus } 21 \% \text { no placebo } \\
(n=47)\end{array}$ \\
\hline
\end{tabular}

Fonte: Autores.

Na pesquisa de Fleury-Teixeira et al. (2019) foram considerados dados de 18 participantes com TEA e idade entre 6 e 17 anos ( $72 \%$ do sexo masculino e $28 \%$ do sexo feminino). Entres os 15 participantes que permaneceram no estudo, foram analisados os efeitos do uso compassivo de Cannabis sativa enriquecida com extrato de CBD (CE) (proporção de CBD/THC de 75/1) no período de 6 a 9 meses. Foi verificado melhoria de mais de 30\% em 80\% dos pacientes nos seguintes parâmetros: transtorno de déficit de atenção e hiperatividade, distúrbios do sono e convulsões. No controle de crises em pacientes epiléticos 
$(\mathrm{n}=5)$, houve redução de $50 \%$ na frequência das crises em três casos e $100 \%$ nos outros dois casos. De forma geral, houve sinais de melhoria nas outras categorias analisadas: transtornos comportamentais, déficits motores, déficits de autonomia; déficits de comunicação e interação social e déficits cognitivos. Foi observado que $47 \%$ dos pacientes tiveram melhora igual ou superior a $30 \%$ em quatro ou mais categorias de sinais. Outro fator positivo foi a redução na dosagem dos medicamentos neuropsiquiátricos em uso concomitante ao $\mathrm{CE}$, inclusive com descontinuidade em alguns casos. Os efeitos colaterais relatados foram leves e/ou transitórios.

Schleider et al. (2019) analisaram a segurança e eficácia da Cannabis no tratamento do autismo. O período de análise compreendeu os anos de 2015 e 2017 e a amostra inicial englobou 188 pacientes com TEA (média de idade de 12,9 anos; $81,9 \%$ do sexo masculino); sendo a epilepsia $(14,4 \%)$ a comorbidade com maior prevalência nessa população. No tratamento da maior parte dos pacientes foi utilizado óleo de Cannabis (30\% de CBD e 1,5\% de THC). A coleta das informações acerca dos sinais, avaliação global e efeitos adversos foram realizadas em três etapas: (1) antes do tratamento; (2) no mês seguinte ao início e (3) seis meses após início do tratamento. Após a última etapa, principalmente no que refere à ordem comportamental, foi observada melhora significativa dos sinais em $30,1 \%$ dos pacientes; $53,7 \%$ melhora moderada; $6,4 \%$ leve melhora e $8,6 \%$ relataram nenhuma mudança em sua condição. Os sinais com maior prevalência de melhora foram, na seguinte ordem: inquietação, raiva, distúrbios do sono, agitação, ansiedade, convulsões, constipação e problemas de digestão. Por outro lado, menor eficácia foram percebidos nos sinais relacionados à: déficit cognitivo, distúrbios de fala, incontinência, mobilidade limitada, apetite aumentado e falta de apetite. Em $25,2 \%$ dos pacientes foram notados efeitos colaterais, como inquietação $(6,6 \%)$.

Outro ponto analisado por Schleider et al. (2019) foi o aumento no número de pacientes que relatavam ter boa qualidade de vida (de 31,3\% para 66,8\%). Os autores (2019) também ressaltam que, a adesão de mais de 80\% dos pacientes ao tratamento indica satisfação com o tratamento, especialmente no que tange aos aspectos comportamentais.

Nos estudos anteriormente citados (Barchel, et al., 2018; Fleury-Teixeira, et al., 2019; Schleider, et al., 2019) não foram utilizadas comparações com grupo controle. Em contrapartida, no ensaio clínico duplo-cego randomizado realizado Aran et al. (2021), 150 participantes foram designados aleatoriamente para três tipos de tratamento: (1) placebo oral; (2) extrato da planta inteira em uma proporção de CBD/THC de 20/1 e (3) CBD purificado e THC na mesma proporção. Com base na escala Clinical Global Impression-Improvement (CGI-I, desfecho primário), foi demostrado o potencial do CBD em diminuir os comportamentos agressivos associados ao TEA em $49 \%$ extrato da planta inteira contra $21 \%$ com placebo. $\mathrm{Na}$ aplicação da Social Responsiveness Scale (SRS-2, desfecho secundário), a pontuação média melhorou 3,6 pontos no placebo contra 14,9 pontos no extrato de planta inteira e 8,2 pontos nos canabinoides puros. Na comparação entre os dois compostos canabinoides, não houve vantagens claras do pressuposto efeito sinérgico de terpenos, flavonoides e canabinoides encontrados no extrato da planta inteira. Em relação aos efeitos adversos, não foram relatados eventos graves.

\section{Discussão}

Para o Transtorno do Espectro Autista (TEA), ainda não existe um tratamento capaz de melhorar de maneira abrangente as três áreas com déficits funcionais: desenvolvimento cognitivo, comportamental e interação social; de forma que os medicamentos tradicionalmente utilizados (antipsicóticos atípicos, inibidores seletivos da recaptação da serotonina, ansiolíticos e estimulantes) atuam em sinais específicos, com uma eficácia limitada e, em geral, com efeitos colaterais que prejudicam a adesão ou continuidade do plano terapêutico. Nesse sentido, o uso do canabidiol (CBD), seja puro ou em extrato, se torna uma opção promissora considerando que os efeitos colaterais são leves e/ou transitórios, a citar os principais: sonolência e falta de apetite (Barchel, et al., 2018; Fleury-Teixeira, et al., 2019; Schleider, et al., 2019, Aran, et al., 2021).

A partir do uso do CBD, as pesquisas analisadas mostram significativa melhora nos aspectos comportamentais, 
apresentando melhora no estudo placebo-comparativo (Aran, et al., 2021) de $28 \%$ no comportamento disruptivo (diferença entre dados da extrato da planta inteira versus placebo), até a melhora de mais de $90 \%$ nas categorias de inquietação e ataques de raiva (Schleider, et al., 2019).

De forma geral, os participantes mostraram ter melhoras de todos os sinais associados ao TEA considerados em cada estudo. A autonomia em na população autista é prejudicada pela presença dos déficits funcionais. Ainda que tenha sido relatada melhora menos significativa em relação as outras comorbidades analisadas, o tratamento com CBD mostra melhoras de $10 \%$ (Fleury-Teixeira, et al., 2019) no déficit de autonomia até melhora de 16,5\% na capacidade de se vestir e tomar banho de forma independente (Schleider, et al., 2019).

Enquanto aspectos comportamentais, podem ser melhorados também com o uso de psicotrópicos; o déficit de interação social pouco melhora com uso de tais medicamentos. Em contrapartida, a terapia com CBD mostrou melhora de até 25\% (Fleury-Teixeira, et al., 2019) nesse aspecto. É importante esclarecer que não se pretende no presente estudo, a simples comparação dos resultados atingidos em cada pesquisa; pois, as metodologias empregadas diferem entre si, além do tamanho da amostra, formulações e doses aplicadas. Uma vez que esses pacientes já utilizam outros medicamentos, deve-se considerar a possibilidade de interações medicamentosas, especialmente com antipsicóticos, como apontado por Fleury-Teixeira et al. (2019), havendo necessidade de maior atenção quanto a administração dos medicamentos em uso.

Em todos os artigos analisados, devido à natureza observacional, cita-se como limitação a subjetividade e a falta de uma ferramenta para avaliação objetiva dos resultados, limitando-se aos relatos dos cuidadores. Com exceção do estudo de Aran et al. (2021), outra limitação é a falta de um grupo controle para comparação. Nesse sentido, Schleider et al. (2019) ressaltam não poder ser estabelecida causalidade entre a terapia com Cannabis e a melhora no bem-estar dos pacientes; considerando, também, que tais variáveis subjetivas podem ser enviesadas pela opinião dos pais/cuidadores.

\section{Considerações Finais}

Considerando que não há tratamento farmacológico estabelecido para o Transtorno do Espectro Autista, especialmente no que concerne aos sinais nucleares, e que os avanços na área desde a década 50 têm sido relativamente modestos; o uso de medicamentos alternativos tem sido alvo de pesquisas, com interesse crescente no tema ao longo dos últimos anos. Ressalta-se, então, a necessidade de estudos que contribuam com entendimento do mecanismo de ação do CBD, seus benefícios e efeitos colaterais no tratamento do TEA, principalmente a longo prazo.

De forma geral, o uso do CBD tem impacto positivo na redução da ansiedade, agressividade e inquietação; podendo haver benefícios nos casos refratários de distúrbio de comportamento, que incluem agitação, distúrbio do sono e heteroagressividade.

Independente da escolha farmacológica, a melhora dos sinais auxilia na continuidade do plano terapêutico, que deve envolver uma equipe multidisciplinar. Nesse sentido, o tratamento precoce, com a regulação dos sinais traz benefícios importantes ao paciente, uma vez que há um impacto direto sob sua qualidade de vida, com foco na independência do indivíduo afetado. Recomenda-se que sejam realizados estudos clínicos randomizados robustos que padronizem variáveis importantes para detecção de efeitos adversos, titulação de doses e duração dos tratamentos. Neste contexto, novos estudos são necessários para avaliar a eficácia e segurança destes novos alvos farmacológicos para o tratamento do TEA.

\section{Referências}

Aran, A., Harel, M., Cassuto, H., Polyansky, L., Schnapp, A., Wattad, N., Shmueli, D., Golan, D., \& Castellanos, F. X. (2021). Cannabinoid treatment for autism: a proof-of-concept randomized trial. Molecular autism, 12(1), 6. 10.1186/s13229-021-00420-2

Arberas, C., \& Ruggieri, V. (2019). Autism. Genetic and biological aspects. Medicina (B Aires). 79(Suppl 1):16-21. Spanish. 
Barchel, D., Stolar, O., De-Haan, T., Ziv-Baran, T., Saban, N., Fuchs, D. O., Koren, G., \& Berkovitch, M. (2019). Oral cannabidiol use in children with Autism Spectrum Disorder to treat related symptoms and co-morbidities. Frontiers in Pharmacology, 9, 1521. doi:10.3389/fphar.2018.01521

Chakrabarti, B., Persico, A., Battista, N., \& Maccarrone, M. (2015). Endocannabinoid Signaling in Autism. Neurotherapeutics: The Journal of the American Society for Experimental NeuroTherapeutics, 12(4), 837-847. doi:10.1007/s13311-015-0371-9

Cooray, R., Gupta, V., \& Suphioglu, C. (2020). Current aspects of the endocannabinoid system and targeted the and cbd phytocannabinoids as potential therapeutics for Parkinson's and Alzheimer's Diseases: a Review. Molecular Neurobiology, 57(11), 4878-4890. doi:10.1007/s12035-020-02054-6

Devinsky, O., Cilio, M. R., Cross, H., Fernandez-Ruiz, J., French, J., Hill, C., Katz, R., Di Marzo, V., Jutras-Aswad, D., Notcutt, W. G., Martinez-Orgado, J., Robson, P. J., Rohrback, B. G., Thiele, E., Whalley, B., \& Friedman, D. (2014). Cannabidiol: pharmacology and potential therapeutic role in epilepsy and other neuropsychiatric disorders. Epilepsia, 55(6), 791-802. doi:10.1111/epi.12631

Estrela, C. (2018). Metodologia Científica: Ciência, Ensino, Pesquisa. Editora Artes Médicas. Arte Med: (3a ed,), p.707.

Ferreira-Junior, N. C., Campos, A. C., Guimarães, F. S., Del-Bel, E., Zimmermann, P., Brum Junior, L., Hallak, J. E., Crippa, J. A., \& Zuardi, A. W. (2020). Biological bases for a possible effect of cannabidiol in Parkinson's disease. Revista Brasileira de Psiquiatria (Sao Paulo, Brazi: 1999), 42(2), 218-224. doi:10.1590/1516-4446-2019-0460

Fleury-Teixeira, P., Caixeta, F. V., Ramires da Silva, L. C., Brasil-Neto, J. P., \& Malcher-Lopes, R. (2019). Effects of CBD-Enriched Cannabis sativa Extract on Autism Spectrum Disorder Symptoms: An observational study of 18 participants undergoing compassionate use. Frontiers in Neurology, 10 , 1145. doi:10.3389/fneur.2019.01145

Hollander, E., Dolgoff-Kaspar, R., Cartwright, C., Rawitt, R., \& Novotny S. (2001). An open trial of divalproex sodium in autism spectrum disorders. Journal of Clinical Psychiatry, 62(7):530-4. doi:10.4088/jcp.v62n07a05

Kis, B., Ifrim, F. C., Buda, V., Avram, S., Pavel, I. Z., Antal, D., Paunescu, V., Dehelean, C. A., Ardelean, F., Diaconeasa, Z., Soica, C., \& Danciu, C. (2019). Cannabidiol-from plant to human body: a promising bioactive molecule with multi-target effects in cancer. International Journal of Molecular Sciences, 20(23), 5905. doi:10.3390/ijms20235905

Linartevichi, V., Cury, R., \& Nascimento, F. (2021). Possível utilização de canabinoides na Doença de Alzheimer: uma revisão. Fag Journal of Health, 3(2), 195-199. doi:10.35984/fjh.v3i2.365

Maneeton, N., Maneeton, B., Putthisri, S., Woottiluk, P., Narkpongphun, A., \& Srisurapanont, M. (2018). Risperidone for children and adolescents with autism spectrum disorder: a systematic review. Neuropsychiatric Disease and Treatment, 14, 1811-1820. doi:10.2147/NDT.S151802

Mottron, L., \& Bzdok, D. (2020). Autism spectrum heterogeneity: fact or artifact? Molecular Psychiatry, 25(12), 3178-3185. doi:10.1038/s41380-020-0748-y

Nikolov, R., Jonker, J., \& Scahill, L. (2006). Autistic disorder: current psychopharmacological treatments and areas of interest for future developments. Brazilian Journal of Psychiatry, 28 Suppl 1:S39-46. Portuguese. doi:10.1590/s1516-44462006000500006

Nunes, T. P., Nunes, L. P., Silva, A. N. A., Pirovani, B. O., Morábito , M. J. S. D., de Morais, L. A., Gruba, A. S., \& Nunes, G. P. (2021). Effects adverse of treatment with selective inhibitors of serotonin recaptation on the fetus and neonatals of mothers with gestational depression: A critical review. Research, Society and Development, 10(4), e11210413937. doi:10.33448/rsd-v10i4.13937

Oliveira, C. R. de A., \& Souza, J. C. (2021). Neurobiologia do autismo infantil. Research, Society and Development, 10 (1), e11910111495. 10.33448/rsdv10i1.11495

Pacher, P., \& Kunos, G. (2013). Modulating the endocannabinoid system in human health and disease--successes and failures. The FEBS Journal, 280(9), 1918-1943. doi:10.1111/febs.12260

Pretzsch, C. M., Voinescu, B., Mendez, M. A., Wichers, R., Ajram, L., Ivin, G., Heasman, M., Williams, S., Murphy, D. G., Daly, E., \& McAlonan, G. M. (2019). The effect of cannabidiol (CBD) on low-frequency activity and functional connectivity in the brain of adults with and without autism spectrum disorder (ASD). Journal of Psychopharmacology (Oxford, England), 33(9), 1141-1148. doi:10.1177/0269881119858306

Reddihough, D. S., Marraffa, C., Mouti, A., O'Sullivan, M., Lee, K. J., Orsini, F., Hazell, P., Granich, J., Whitehouse, A., Wray, J., Dossetor, D., Santosh, P., Silove, N., \& Kohn, M. (2019). Effect of fluoxetine on obsessive-compulsive behaviors in children and adolescents with autism spectrum disorders: a randomized clinical trial. JAMA, 322(16), 1561-1569. doi:10.1001/jama.2019.14685

Reece, A. S., \& Hulse, G. K. (2019). Impacts of cannabinoid epigenetics on human development: reflections on Murphy et. al. 'cannabinoid exposure and altered DNA methylation in rat and human sperm' epigenetics 2018; 13: 1208-1221. Epigenetics, 14(11), 1041-1056. doi:10.1080/15592294.2019.1633868

Rubim, A. L., \& Hora, A. F. L. T. da. (2020). Social skills training possibilities for adults with autism spectrum disorder: a systematic review. Research, Society and Development, 9(7), e414974287. doi:10.33448/rsd-v9i7.4287

Silva, C. O. da, Oliveira, S. A., Silva, W. C. da, Mendes, R. C., Miranda, L. S. C., Melo, K. C., Silva, R. A. da, Oliveira, T. M. P. de, Oliveira, C. de J. P., \& Santos, M. E. de J. (2020). Benefits in using early intervention in children with autista spectrum disorder (ASD): an integrative review. Research, Society and Development, 9(7), e256972474. doi:10.33448/rsd-v9i7.2474

Silva, W. M. da, \& Coelho, A. T. C. B. (2021). The language acquisition process for children with autistic spectrum disorder: review article. Research, Society and Development, 10(1), e15010111584. doi:10.33448/rsd-v10i1.11584

Starowicz, K., \& Finn, D. P. (2017). Cannabinoids and pain: sites and mechanisms of action. Advances in Pharmacology, 80:437-475. doi:10.1016/bs.apha.2017.05.003

Schleider, L. B., Mechoulam, R., Saban, N., Meiri, G., \& Novack, V. (2019). real life experience of medical cannabis treatment in autism: analysis of safety and efficacy. Science Report, 9(1):200. doi:10.1038/s41598-018-37570-y 